\title{
In search of European excellence
}

\author{
F. Gros and G. P. Tocchini-Valentini
}

\begin{abstract}
What should be the priorities for supporting life-science research in Europe? This article, an upshot of an initiative by Commissioner Ruberti of the European Union, addresses the question.
\end{abstract}

THE new Framework Programme of the European Union, which will provide funds for scientific research and technological development from 1994 to 1998 , is the subject of intense debate. The programme is proposed by the European Commission but then has to be decided upon jointly by the Council, made up of the science ministers of the member states, and the European Parliament. The result will be the definition of the scientific and technological objectives of the Union for the next five years. Support will be provided for research, technological development and demonstration programmes; for scientific cooperation with countries outside Europe and international organizations; for programmes to encourage the application of the results of research to practical ends; and for encouraging the training of scientists and their mobility within Europe.

Whatever the point of view from which one examines the specific programmes within the Framework, the long-term aims, for example the improvement of industrial competitiveness in Europe, cannot be achieved if the scientific work is not evaluated and supported according to the criterion of excellence. Commissioner Antonio Ruberti of the European Union, who has responsibility for science, research and development, has been keen to solicit independent scientific advice. To this end, he has appointed advisors to provide him with their views on research trends in the life sciences (F. Gros), high-energy physics (C. Rubbia) and applied mathematics and complex systems (I. Prigogine). As part of this process we chaired a meeting of 25 scientists with expertise in biological, biomedical and biotechnological research in Brussels at the end of October 1993. The object was to arrive at specific recommendations that might form part of the basis for the Commission's deliberations. This article is the summary of our discussions*.

\section{The science}

The following areas of research particulary require action by concerted means.

*This report appears under the names of the co-chairmen of the Brussels meeting, F. Gros and G. P. Tocchini-Valentini, but was contributed to by all participants. They were: M. Bishop. M. Buckingham, A. Capron, L. L. Cavalti-Sforza, S. Cusak, A. Falaschi, R. B. Flavell, A. Garcia-Bellido, M. GrunbergManago, P. Gruss, K. Hoffman, R. Huber, M. Kafatos, U. Laemmli, G. Nicolis, R. Rajewsky, P. Rigby, J. Schell, P. Slonimski, D. Von Wettstein, J. Weissenbach, R. Williamson and A. Xavier.
We believe that they meet the criteria of the fourth Framework, in that they will serve to increase the competitiveness of European industry, and will also contribute directly to improvements in the health of European societies.

1. Structural biology. One of the main problems in modern life-science research is that we do not know enough about the relationship between primary structure, (nucleic acid or protein sequence), and the tertiary structures of biologically active macromolecules, and we know even less about the quaternary structures of the multi-subunit complexes that mediate most biological activities. So we cannot yet accurately predict biological function from structural motifs.

Advances in biological structure determination mean that data collection is now relatively rapid, and that it is possible to work out the structures of protein-protein complexes and of complexes between proteins and other macromolecules (DNA, RNA, carbohydrates and lipids). Understanding the structural basis of such complex formation is essential to the discovery and refinement of new chemical entities which either modulate or interfere with complex formation, and is thus of direct relevance to the pharmaceutical industry. Studies of RNA, which can act as both a genetic message and an enzyme, will be of importance and interest, particularly given the role of RNA in the evolution of life.

This research will require the continued application of X-ray crystallography, nuclear magnetic resonance spectroscopy, electron microscopy and confocal microscopy, and work to improve the resolution of these techniques and the size of structure that they can analyse will be crucial. These technical developments will allow more detailed studies of sub-cellular structures - chromosomes, spliceosomes and replisomes, for example - with further implications for both biological understanding and health-care provision.

2. Developmental biology. Developmental biology deals with the mechanisms by which the three-dimensional structure of the organism and its many and complex functions are derived from the fertilized egg. Central to understanding this process is knowledge of how the body plan of the embryo is first established, how the information in that body plan is subsequently interpreted, and how morphogenetic processes (for example cell-cell inter- actions and cell migration) give rise to the final organism. It will also be of great importance to study the development of particular structures, for example the brain. Studies of the regulation of gene activity in both time and space are relevant to all of these issues. Our view is that research in this area under the Framework Programme should be confined to a few experimentally tractable model organisms.

Understanding how organisms are constructed, how cells are instructed as to what they should be, and where they should be, is essential to finding out how these processes malfunction in disease. Such knowledge is also highly relevant to research into regenerative processes in adult organisms, for example wound healing and the repair of the nervous system. Work of this sort is particularly timely because of the advent of technologies for manipulating the genome of intact vertebrates and because of the availability of techniques that enable us to identify the molecular signals that induce cellular responses. Of particular interest will be the identification of such triggering molecules, the receptors with which they interact, and the internal cascades of responses that ultimately regulate the activities of transcription factors and lead to adaptive patterns of gene expression. These events are central to the modulation of differentiation and in determining normal as well as abnormal development. The new technologies involved represent the biological revolution of the 1990s, which will be equivalent in its effects to the revolution of the 1980 s based on recombinant DNA technology.

These advances will allow us both to model and to understand complex disease pathologies. They are thus of direct relevance to health care and to the needs of the pharmaceutical industry, as they will lead to the availability of test-beds for the development of new drugs and gene therapies.

3. Genome research. The study of the human genome is central to improved understanding of the genetic basis of inherited diseases such as cystic fibrosis and thalassaemia, as well as complex clinical conditions such as cancer, heart disease and Alzheimer's disease. Structural analysis of genomes must go hand in hand with studies of the mechanisms of gene function. In this regard, in addition to the human genome it will be necessary to 
concentrate on a few carefully chosen model systems (Bacillus subtilis, yeast, Arabidopsis, Drosophila and the mouse). The mouse is of crucial importance in that, at the moment, it is the only practicable model for human disease pathology. Our view is that comparative genome mapping has a central role in maximizing the efficiency with which the results of genome analyses are converted into biologically useful information.

4. Health and agricultural research. In the health sector, newly identified molecules and cellular processes will surely become essential tools, so long as they can be fully developed in the context of the physiology of animal and human diseases. The design and use of animal models for gene targeting (so-called 'gene knockout') experiments is now indispensable to strategies for disease control. Moreover, the development of new vectors will be essential for most future applications of somatic gene therapy, a potential route for treating inherited diseases, cancer and possibly AIDS. Particular attention should be given to work on infectious diseases, especially vaccine development where more concerted European efforts are needed. Other notable areas that we should continue to address are the basic biology of cancer, the pathogenesis of autoimmune diseases, the study of the brain in health and disease, and the development of gene therapy.

Both agriculture and our environment will benefit from the possibility that we will have a complete molecular understanding of plant biology. Of particular priority will be studies of the physiological responses to stress and pathogenesis, the metabolism of the storage products that make up the chemical diversity of the biomass, and the modulation of heterologous gene expression. The introduction of specially designed genes will pave the way for the efficient use of plant processes for the production of specified end products.

A selective programme of plant science research, bringing together those who create the technology and those who need to use it, would be of great value in addressing agricultural, industrial and environmental issues within the Union. Much of this research will be applied to creating environmentally sustainable crop systems and new options for integrated production and processing chains. New substrates for the food industry, improved processes, and assays for determining quality and quantity of food attributes will become available.

\section{The infrastructures}

The efficient pursuit of life-science research in Europe depends upon the provision of certain infrastructures that are beyond the means and competence of national authorities and private initiative. These facilities must be responsive to user needs, and there must be mechanisms for ensuring their quality in that respect.

1. Genetic archives and stock centres. The ability to subject any gene to specific mutation opens the way to a revolution in our understanding of the normal function of genes and their malfunction in pathological processes. But it is beyond the means of even the best funded national laboratories to maintain the stocks of all of the mutant animals that they will generate. Moreover, much of the benefit of this technology will come from the interbreeding of such mutants, to create organisms with multiple defects. It will also be essential for researchers to be able to re-access mutants that were made for a particular purpose, for use in research that could not have been predicted at the time that the mutant was first made. So we need to ensure that all such mutants are freely available to researchers within the Union and that the information available to each generation of researchers is not lost.

We therefore propose that the community should support the best initiatives aiming to establish European repositories for three model organisms relevant to modern genetics, biomedicine and agriculture.

A repository of mouse (and rat) mutants will be indispensable for work on gene interactions, which is itself vital for studies of the diseases (such as cancer and coronary heart disease) that are the main causes of illness and death in the Union. The activities of this repository should, to some extent, be modelled on and be complementary to those of the Jackson Laboratory in Maine in the United States. Likewise, a European stock of Drosophila mutants is desirable, because this organism has and will be the source of a great deal of information on gene structure and function, as are European plant repositories (for reasons that are identical in principle but different in details).

2. Bioinformatics. The provision of central facilities for biological information storage, retrieval and analysis also transcends national boundaries. The availability of up-to-date and professionally curated data libraries, as exemplified by the data library at the European Molecular Biology Laboratory, is essential for all of European biology; the accumulation of genomic sequences and protein-structure information will deepen this need. Core European databases would have to be developed in such a way as to prevent duplication of effort and waste of funds. The Union should assure, and possibly expand, the provision of such strategic services, providing the necessary funds to the centre or centres that creates and maintains high standards and user satisfaction. Furthermore, there is a clear need for a European database of protein structure and function. Union funds should support the development and integration of such core services with other, higher order, specialized databases, thus ensuring coordination and the broad distribution of all sources of biological data through the network of national nodes for bioinformatic users.

The efficiency of European endeavours in this area will depend on two features, centralization and synergy. On the one hand, the existence of single entry points for each service will save resources and simplify the submission of information and subsequent access to it. On the other, sharing resources through a network in which the minimum number of data providers serves the largest community of users will have synergistic effects.

3. Training and mobility. Schemes for training researchers and encouraging the mobility of scientists within the Union are of great value. In formulating action to be taken under the new Framework Programme, it will be necessary to consider past experience and address new needs. The trans-national twinning or networking of laboratories collaborating on research is crucial and should receive additional support. We should, we believe, return to the flexibility of the SCIENCE programme. In particular, there should not be quotas for different budget items the types of personnel required and details of budget allocation will depend on the nature of the project.

Fellowships for training through research will have to take into account current worldwide trends, for example the lengthening of the post-doctoral period, which in the life sciences is now 3-4 years. Such fellowship programmes should also provide adequate research support to the host laboratory and be set up so as to encourage training in an interdisciplinary setting. We strongly support the idea that the European Union should begin to fund formal courses to prepare young scientists for the use of advanced facilities.

Now that there is a healthy level of mobility of post-doctoral trainees within Europe, the similar needs of young, independent researchers ought to be addressed. We would favour a programme to support excellent young scientists (for, say, seven years) in establishing a research group outside their country of origin. Such an initiative would help create a truly European scientific community and would contribute to the independence of scientists during their most creative years; moreover, by acknowledging the special needs of less favoured regions, it would strengthen the overall cohesion of the European community.

F. Gros is at the Institut Pasteur, 28 rue du Dr Roux, 75724 Paris Cedex 15, France. G. $P$. Tocchini-Valentini is at the Istituto di Biologia Cellulare, Viale Marx 43, 00137 Roma, Italy. 